\title{
Arterial stiffness shown by the cardio-ankle vascular index is an important contributor to optic nerve head microcirculation
}

\author{
Tomoaki Shiba $^{1} \cdot$ Mao Takahashi $^{2}$ (D) Tadashi Matsumoto $^{1} \cdot$ Kohji Shirai $^{3} \cdot$ \\ Yuichi Hori $^{1}$
}

Received: 23 May 2016 /Revised: 6 September 2016 / Accepted: 5 October 2016 / Published online: 14 October 2016

(C) The Author(s) 2016. This article is published with open access at Springerlink.com

\begin{abstract}
Propose The purpose of this study was to determine whether there were significant correlations between the pulse waveform in the capillary area of the optic nerve head (ONH) microcirculation shown by laser speckle flowgraphy (LSFG) and parameters of the systemic condition, especially the cardio-ankle vascular index (CAVI).

Method We studied 130 men (ages $60.5 \pm 10.9$ years) who visited the Vascular Function Section of the Department of Cardiovascular Center of Toho University Sakura Medical Center. We evaluated the skew and blowout time (BOT) which are parameters of pulse waveform analyses - using LSFG in the capillary area of the ONH for each patient. The CAVI, the E/e' ratio as the measure of diastolic left ventricular function, and the mean intima-media thickness (IMT) were evaluated as systemic parameters. We performed a Pearson's correlation analysis and a multiple regression analysis to determine independent factors for skew and BOT.

Results Heart rate, spherical refraction, and the CAVI (standard regression $=0.18, t=2.61, p=0.01$ ) were revealed as factors contributing independently to the skew by multiple regression analysis. Heart rate, the CAVI (standard regression $=-0.27, t=-3.92, p=0.0002$ ), the urinary albumin concentration, the mean IMT, spherical refraction, body mass
\end{abstract}

Mao Takahashi

takahashi-04@sakura.med.toho-u.ac.jp

1 Department of Ophthalmology, School of Medicine Toho University, Tokyo, Japan

2 Cardiovascular Center, Toho University Sakura Medical Center, 564-1 Shimoshizu, Sakura, Chiba 285-8741, Japan

3 Internal Medicine, Toho University Sakura Medical Center, Sakura, Chiba, Japan index and pulse pressure were revealed as factors contributing independently to the BOT by multiple regression analysis. Conclusion The CAVI was demonstrated to be an independent factor contributing to both skew and BOT in the capillary area of the ONH. Our findings clarified that large arterial function shown by the CAVI contributes to smooth hemodynamics of microcirculation, shown by LSFG.

Keywords Cardio-ankle vascular index - Ocular circulation . Laser speckle flowgraphy $\cdot$ Pulse waveform analysis .

Arteriosclerosis

\section{Introduction}

Systemic arteriosclerosis is an important contributing factor to cardiovascular disease and cerebrovascular disease, accounting for much of the mortality due to these diseases [1]. An arterial stiffness parameter, the cardio-ankle vascular index (CAVI), was developed as a marker related to arteriosclerosis including that of the aorta, femoral artery, and tibial artery [2]. The CAVI is theoretically independent of blood pressure, because the CAVI is measured from the stiffness parameter, $\beta$, which was proposed by Hayashi et al. [3] The CAVI is based on the variance of the arterial pressure that is required to change the vascular diameter.

There are also several reports of relationships between systemic arteriosclerosis and ocular diseases, such as retinal vein occlusion- and age-related macular degeneration [4-9]. It is thus clear that important risk factors for cardiovascular disease, cerebrovascular disease and retinal disease overlap. This concept is supported by the finding that the ocular microcirculation provides a window for detecting changes in microvasculature related to the development of cardiovascular diseases [10]. 
Laser speckle flowgraphy (LSFG), a noninvasive quantitative method of determining ocular blood flow [11, 12], is based on the changes in the speckle pattern of laser light reflected from the fundus of the eye [13, 14]. LSFG is dependent on the movement of erythrocytes in the retina, the choroid and the optic nerve head $(\mathrm{ONH})$, and the mean blur rate (MBR) is an indicator of ocular blood flow [15, 16]. In 2008, the LSFG-NAVITM (Softcare Co., Fukuoka, Japan) was approved as a medical apparatus by the Pharmaceuticals and Medical Devices Agency in Japan. Changes in the MBR have various pulse wave patterns that are synchronized with the cardiac cycle.

We have focused on the relationship between the pulse waveform and patients' systemic conditions, and we reported that the pulse waveform in the ONH was significantly correlated with the CAVI, the left ventricular function obtained from echocardiography, and the carotid intima media thickness (IMT) as shown by ultrasonographic imaging, in healthy subjects $[17,18]$. Other researchers clarified that the skew and blowout time (BOT) in the capillary area of the ONH (which are factors derived from pulse waveform analyses) are important predictive factors for normal tension glaucoma and silent brain infarction $[19,20]$. In light of all of these findings, we hypothesized that systemic conditions are affected by the skew and BOT in the capillary area of the ONH.

The purpose of this study was thus to determine whether there are significant correlations between the skew and BOT in the capillary area of the ONH shown by LSFG and parameters of the systemic condition, such as the CAVI, mean IMT and left ventricular diastolic function in patients with a variety of disorders.

\section{Materials and methods}

We analyzed the cases of 154 consecutive men who visited the Vascular Function Section of the Department of Cardiovascular Center of Toho University Sakura Medical Center in the years from April 1, 2007 to December 1, 2012. Patients who had glaucoma, moderate degree of cataract, vitreous and retinal diseases, and those who underwent intraocular surgery were excluded. The patients with the following conditions were excluded because these conditions can affect the CAVI and LSFG measurement: chronic atrial fibrillation had decreased the patient's left ventricular function (ejection fraction $<50 \%$ ), and peripheral arterial disease defined as an ankle-brachial index $<0.9$. In the end, 130 patients whose mean \pm standard division (SD) age was $60.5 \pm 10.9$ years with the range 29 to 83 years met the study criteria. The Institutional Review Board of Toho University Sakura Medical Center approved the protocol of this study, and we began the research after all patients received information on the purpose and possible side effects of the research protocol and signed an informed consent. The procedures used conformed to the tenets of the Declaration of Helsinki.

Echocardiography, CAVI measurement, and LSFG were performed after the patient rested for $10 \mathrm{~min}$ in a quiet, airconditioned room with the temperature maintained at $24^{\circ} \mathrm{C}$. All patients abstained from smoking, alcohol and caffeine for at least $24 \mathrm{~h}$ prior to the measurements. All evaluations were made between 15:00 and 17:00 $\mathrm{h}$ before a meal.

\section{Measurements of echocardiography}

Left ventricular diastolic function was assessed according to the recent consensus guidelines [21, 22] on diastolic function evaluation, measuring the mitral inflow velocities (E-wave) using pulse wave Doppler in the apical four-chamber view. The pulse wave tissue-Doppler velocities were acquired at end-expiration, in the apical four-chamber view, with the sample positioned at the lateral mitral annulus, measuring early diastolic (e' velocity) and calculating the E/e' ratio. The E/e' ratio has been reported to be the single best predictor of the left ventricle diastolic filling pressure [23]. Echocardiography was performed using a commercially available instrument (Vivid 7, GE Healthcare, Tokyo).

\section{Measurements of mean IMT}

High-resolution ultrasonographic imaging of the carotid artery using the B-scan mode was performed using the EUB-8500 ultrasound system (Hitachi, Tokyo) with the probe frequency set to $7.5 \mathrm{MHz}$. The imaging was performed with the patient in the supine position with his head turned slightly away from the sonographer. The procedure involved scanning the near and far walls of the carotid artery every $1 \mathrm{~cm}$ proximal to the carotid bulb in the longitudinal view. The mean IMT was defined as the average of the maximal IMT $1 \mathrm{~cm}$ proximal and $1 \mathrm{~cm}$ distal to the carotid bulb $[24,25]$. The mean IMT of the right side of the carotid artery was used for the data analyses.

\section{Measurement of the CAVI, mean arterial blood pressure, and pulse pressure}

The measurements of the CAVI, blood pressure, and heart rate were performed using the program embedded in the VaSera VS-1000, a vascular screening system (Fukuda Denshi, Tokyo). A detailed explanation of the methods used to measure the CAVI has been reported [2]. Briefly, the brachial and ankle pulse waves were determined with inflatable cuffs with the pressure maintained at $30-50 \mathrm{mmHg}$ to ensure that the cuff pressure had a minimal effect on the systemic hemodynamics. The blood pressure and pulse pressure were 
determined simultaneously [2]. The measurements were made with the patient in the supine position. The mean arterial blood pressure (MABP: $\mathrm{mmHg}$ ) and pulse pressure $(\mathrm{mmHg})$ were determined by the following formulae:

MABP $=$ diastolic blood pressure $+($ systolic blood pressure-diastolic blood pressure $) / 3$.

Pulse pressure $=$ systolic blood pressure-diastolic blood pressure.

\section{LSFG measurements}

We calculated the parameters using LSFG Analyzer software (v.3.0.47, Softcare Co., Fukuoka, Japan). The software program then separated out the vessels using the automated definitive threshold and divided the $\mathrm{ONH}$ into the vessel area and the capillary area. The details of the determination of the LSFG measurements from fundus images were described previously $[16,26]$. In short, LSFG uses the mean blur rate (MBR) as an indicator of the relative velocity of erythrocytes. The skew and BOT (as items of the pulse waveform analyses) were calculated. For the evaluation of the capillary area of the $\mathrm{ONH}$, a circle was set surrounding the ONH. First, $118 \mathrm{MBR}$ images (118 frames) were recorded from the circle and the rectangular area within a 4-s period tuned to the cardiac cycle. A gray-scale map of the still images was then made by averaging the MBR images. On the analysis screen, the pulse wave of the changing MBR values, which corresponded to each cardiac cycle, was obtained. The analysis of the screen, which is normalized to one pulse, is then displayed, and the analysis of the pulse waveform and average MBR are made on this screen.

A similar explanation of the BOT values were obtained from the waveform analysis [16, 19, 27, 28]. Skew quantifies the asymmetry of the waveform distribution, varying with the bias of the waveform shape. Specifically, the skew equals zero if the waveform is completely symmetrical, and the skew becomes positive or negative if the waveform is distributed leftward or rightward of the center point of the waveform [16].

Only the data from the right eye were used for the analysis.

\section{Measurements of laboratory profile}

The laboratory profile of each patient was comprised of the determination of the following: glycated hemoglobin A1c (HbAlc: \%), homeostasis model assessment of insulin resistance (HOMA-IR), apolipoprotein-A $1(\mathrm{mg} / \mathrm{dL})$, apolipoprotein-B $(\mathrm{mg} / \mathrm{dL})$, hematocrit $(\%)$, platelet count $(\times 104 / \mu \mathrm{L})$, cystatin $\mathrm{C}(\mathrm{mg} / \mathrm{L})$ and urinary albumin concentration (UAC: $\mathrm{mg} / \mathrm{L}$ ) as an indicator of kidney function, and the total cholesterol $(\mathrm{mg} / \mathrm{dL})$ obtained from fasting morning blood and urine samples. The HbAlc is expressed based on the scale of the National Glycohemoglobin Standardization Program. HOMA-IR $=$ fasting insulin $(\mathrm{IU} / \mathrm{ml}) \times$ fasting blood glucose $(\mathrm{mmol} / \mathrm{L}) / 22.5$ [29]. Blood and urine samples were centrifuged immediately after collection at $4,000 \mathrm{~g}$ for $10 \mathrm{~min}$, and the supernatant was frozen immediately in polypropylene tubes and stored at $-80{ }^{\circ} \mathrm{C}$ until its use in assays.

\section{Measurements of other systemic parameters and other ocular parameters}

The following parameters were measured: body mass index (BMI: $\mathrm{kg} / \mathrm{m}^{2}$ ), spherical refraction (diopters) assessed with the TONOREF 2 system (NIDEK, Aichi, Japan), ocular perfusion pressure (OPP, $\mathrm{mmHg}$ ), and heart rate (beats per min). The OPP was defined as: (2/3MABP) - intraocular pressure. The intraocular pressure was obtained by applanation tonometry.

\section{Statistical analyses}

Data are presented as the means \pm SD for the continuous variables. Pearson's correlation coefficients and multiple regression analysis were used to determine independent factors for skew and BOT values in the capillary area of the ONH.

We considered $p$-values $<0.05$ statistically significant. The StatView program ver. 5.0 (SAS, Cary, NC) was used for the statistical analyses.

\section{Results}

The results of the characteristics of all patients are shown in Table 1. Eighty-two of the 130 (63\%) patients had hypertension, and 32 of the 130 patients $(25 \%)$ had diabetes mellitus. The mean \pm SD CAVI was $8.9 \pm 1.1$; the mean \pm SD skew was $13.1 \pm 1.9$, and the mean \pm SD BOT was $46.7 \pm 4.7$. The results of the Pearson's correlation analyses between the skew and the BOT in the capillary area of the $\mathrm{ONH}$ and all parameters are shown in Table 2.

Skew was significantly correlated with age $(r=0.49$, $p<0.0001)$, BMI $(r=-0.23, p=0.008)$, pulse pressure $(r=0.32, p=0.0002)$, spherical refraction $(r=0.32, p=$ $0.0002)$, heart rate $(r=-0.59, p<0.0001), \mathrm{HbA} 1 \mathrm{c}$ $(r=0.21, p=0.02)$, hematocrit $(r=-0.28, p=0.001)$, mean IMT $(r=0.36, p<0.0001)$, the E/e' ratio $(r=0.31$, $p=0.0004)$ and the CAVI $(r=0.39, p<0.0001)$. Cystatin C 
Table 1 Characteristics of all patients $(n=130)$

\begin{tabular}{|c|c|}
\hline \multicolumn{2}{|l|}{ Characteristics } \\
\hline Age (yrs) & $60.5 \pm 10.9$ \\
\hline BMI $\left(\mathrm{kg} / \mathrm{m}^{2}\right)$ & $25.4 \pm 3.7$ \\
\hline Hypertension (\%) & 82 \\
\hline Diabetes mellitus (\%) & 32 \\
\hline MABP (mmHg) & $92.6 \pm 11.8$ \\
\hline Pulse pressure (mmHg) & $55.8 \pm 13.2$ \\
\hline Ocular perfusion pressure $(\mathrm{mmHg})$ & $49.1 \pm 8.0$ \\
\hline Heart rate (beat per minute) & $65.8 \pm 9.7$ \\
\hline $\operatorname{HbA1c}(\%)$ & $6.1 \pm 0.8$ \\
\hline HOMA-IR & $1.9 \pm 1.3$ \\
\hline Apolipoprotein-A1 (mg/dL) & $135.8 \pm 21.5$ \\
\hline Apolipoprotein-B (mg/dL) & $92.2 \pm 20.1$ \\
\hline Hematocrit $(\%)$ & $42.3 \pm 4.0$ \\
\hline Platelet counts $\left(\times 10^{4} / \mu \mathrm{L}\right)$ & $22.0 \pm 5.5$ \\
\hline eGFR (ml/min/1.73 m²) & $69.8 \pm 14.2$ \\
\hline CAVI & $8.9 \pm 1.1$ \\
\hline Range & $6.3-11.8$ \\
\hline \multicolumn{2}{|l|}{ Echo graphical characteristics } \\
\hline Mean IMT (mm) & $0.80 \pm 0.15$ \\
\hline Plaque score & $5.2 \pm 4.8$ \\
\hline $\mathrm{E} / \mathrm{e}^{\prime}$ ratio & $10.7 \pm 3.4$ \\
\hline \multicolumn{2}{|l|}{ LSFG measurements } \\
\hline Skew-Capillary & $13.1 \pm 1.9$ \\
\hline Skew-whole of the ONH & $12.3 \pm 1.8$ \\
\hline BOT-Capillary & $46.7 \pm 4.7$ \\
\hline BOT-whole of the ONH & $49.2 \pm 4.3$ \\
\hline \multicolumn{2}{|l|}{ Anti-hypertensive medicine } \\
\hline Calcium channel blocker $(\%)$ & 53.1 \\
\hline ACEI $(\%)$ & 6.2 \\
\hline $\mathrm{ARB}(\%)$ & 31.5 \\
\hline Diuretics (\%) & 5.4 \\
\hline$\beta$-blocker/ $\alpha$ - $\beta$ blocker $(\%)$ & 13.3 \\
\hline
\end{tabular}

Data given as mean $\pm \mathrm{SD}$ or $\%$

$A C E I$ angiotensin-converting enzyme inhibitor, $A R B$ angiotensinII receptor blocker, $B M I$ body mass index, $B O T$ blowout time, $C A V I$ cardio-ankle vascular index, eGFR estimated glomerular filtration rate, $\mathrm{HbAlc}$ glycated hemoglobin A1c, HOMA-IR homeostasis model assessment of insulin resistance, $I M T$ intima-media thickness, $L S F G$ laser speckle flowgraphy graphy, $M A B P$ mean arterial blood pressure

tended to be correlated with the skew but did not reach significance $(r=0.15, p=0.09)$.

The BOT was significantly correlated with age $(r=-0.64$, $p<0.0001)$, BMI $(r=0.27, p=0.002)$, pulse pressure $(r=-0.38, p<0.0001)$, spherical refraction $(r=-0.29$, $p=0.0008)$, heart rate $(r=0.45, p<0.0001), \mathrm{HbA} 1 \mathrm{c}$ $(r=-0.22, p=0.01)$, hematocrit $(r=0.30, p=0.0006)$, cystatin $\mathrm{C}(r=-0.19, p=0.03), \mathrm{UAC}(r=-0.18, p=0.04)$, mean IMT $(r=-0.46, p<0.0001)$, the E/e' ratio $(r=-0.32, p=0.0002)$ and the CAVI $(r=-0.51, p<0.0001)$.
Table 2 Pearson's correlation analysis between the CAVI and all parameters $(n=130)$

\begin{tabular}{lrl}
\hline & $r$ & $p$ \\
\hline Age & 0.62 & $<0.0001$ \\
BMI $\left(\mathrm{kg} / \mathrm{m}^{2}\right)$ & -0.21 & 0.02 \\
MABP $(\mathrm{mmHg})$ & 0.08 & 0.38 \\
Pulse pressure $(\mathrm{mmHg})$ & 0.29 & 0.0007 \\
Ocular perfusion pressure $(\mathrm{mmHg})$ & 0.10 & 0.27 \\
Heart rate (beat per minute) & -0.03 & 0.73 \\
HbA1c $(\%)$ & 0.24 & 0.007 \\
HOMA-IR & -0.11 & 0.22 \\
Apolipoprotein-A1 $(\mathrm{mg} / \mathrm{dL})$ & 0.05 & 0.55 \\
Apolipoprotein-B $(\mathrm{mg} / \mathrm{dL})$ & -0.10 & 0.28 \\
Hematocrit $(\%)$ & -0.20 & 0.03 \\
Platelet counts $\left(\times 10^{4} / \mu \mathrm{L}\right)$ & -0.26 & 0.002 \\
eGFR (ml/min/1.73 $\left.{ }^{2}\right)$ & -0.27 & 0.002 \\
Mean IMT (mm) & 0.42 & $<0.0001$ \\
Plaque score & 0.37 & $<0.0001$ \\
E/e' ratio & 0.21 & 0.02 \\
Skew-Capillary & 0.39 & $<0.0001$ \\
Skew-whole of the ONH & 0.32 & 0.0002 \\
BOT-Capillary & -0.51 & $<0.0001$ \\
BOT-whole of the ONH & -0.44 & $<0.0001$ \\
& &
\end{tabular}

$B M I$ body mass index, $B O T$ blowout time, $C A V I$ cardio-ankle vascular index, $e G F R$ estimated glomerular filtration rate, $H b A l c$ glycated hemoglobin A1c, HOMA-IR homeostasis model assessment of insulin resistance, IMT intima-media thickness, MABP mean arterial blood pressure

Tables 3 and 4 shows the results of our multiple regression analysis for factors that independently contribute to the skew and BOT in the capillary area of the ONH. The factors contributing independently to the skew $(R=0.78, p<0.0001)$

Table 3 Results of the multiple regression analysis for factors independently contributing to the CAVI, using the blowout time in the capillary $(n=130)$

\begin{tabular}{llll}
\hline Explanatory variable & Standard regression & $t$ & $p$ \\
\hline BOT-Capillary & -0.28 & -3.04 & 0.003 \\
Platelet counts & -0.21 & -2.87 & 0.005 \\
eGFR & -0.16 & -2.10 & 0.04 \\
HbA1c & 0.16 & 2.09 & 0.04 \\
Mean IMT & 0.17 & 2.01 & 0.046 \\
Plaque score & 0.11 & 1.34 & 0.18 \\
BMI & -0.09 & -1.07 & 0.29 \\
Pulse pressure & 0.04 & 0.53 & 0.60 \\
Hematocrit & 0.02 & 0.30 & 0.77 \\
E/e' ratio & -0.01 & -0.15 & 0.88 \\
\hline
\end{tabular}

Objective variable: CAVI. BOT blowout time, $C A V I$ cardio-ankle vascular index, $e G F R$ estimated glomerular filtration rate, $H b A l c$ glycated hemoglobin A1c, IMT intima-media thickness, $R=0.64, p<0.0001$ 
Table 4 Results of the multiple regression analysis for factors independently contributing to the CAVI, using the skew in the capillary $(n=130)$

\begin{tabular}{lcrl}
\hline Explanatory variable & Standard regression & \multicolumn{1}{l}{$t$} & $p$ \\
\hline Platelet counts & -0.22 & -2.18 & 0.005 \\
Mean IMT & 0.21 & 2.54 & 0.01 \\
eGFR & -0.17 & -2.18 & 0.03 \\
HbA1c & 0.17 & 2.17 & 0.03 \\
Skew-Capillary & 0.17 & 2.00 & 0.048 \\
Plaque score & 0.14 & 1.65 & 0.10 \\
BMI & -0.12 & -1.44 & 0.15 \\
Pulse pressure & 0.07 & 0.79 & 0.43 \\
Hematocrit & 0.02 & 0.23 & 0.82 \\
E/e' ratio & -0.002 & -0.03 & 0.98 \\
\hline
\end{tabular}

Objective variable: CAVI, $R=0.63, p<0.0001$

$C A V I$ cardio-ankle vascular index, $e G F R$ estimated glomerular filtration rate, $H b A l c$ glycated hemoglobin A1c, IMT intima-media thickness

were heart rate (standard regression $=-0.49, t=-8.02$, $p<0.0001$ ), spherical refraction (standard regression $=0.24$, $t=3.89, p=0.0002$ ) and CAVI (standard regression $=0.18$, $t=2.61, p=0.01)$. The factors that were contributing independently to the BOT $(R=0.78, p<0.0001)$ were heart rate (standard regression $=0.32, t=5.15, p<0.0001)$, the CAVI (standard regression $=-0.27, t-3.92, p=0.0002)$, UAC (standard regression $=-0.17, t=-2.84, p=0.005$ ), mean IMT (standard regression $=-0.19, t=-2.74, p=0.007)$, spherical refraction (standard regression $=-0.16, t=-2.60, p=0.01$ ), BMI (standard regression $=0.16, t=2.45, p=0.02$ ) and pulse pressure (standard regression $=-0.15, t=-2.23, p=0.0001$ ).

\section{Discussion}

This study was designed to determine whether systemic arterial stiffness shown by the CAVI significantly affects the skew and BOT, which are factors derived from a pulse waveform analysis, in the capillary area of the $\mathrm{ONH}$. In our previous research, we reported that the BOT of the entire $\mathrm{ONH}$ was significantly correlated with age, mean IMT, left ventricular diastolic function, kidney function and the CAVI in the healthy subjects $[17,18,30,31]$. However, in that research we examined only a single correlation to determine the relationship between the BOT and the CAVI [18].

The novel analysis method of LSFG can be used to separate the $\mathrm{ONH}$ into vessel and capillary areas for more detailed analyses of the $\mathrm{ONH}$ microcirculation. It was reported that the skew and BOT values in the capillary area of the ONH are important predictive factors for normal-tension glaucoma and silent brain infarction $[19,20]$.
Based on the above-mentioned findings, we hypothesized that the systemic arterial morphological and functional status also affects the skew and BOT values in the capillary area of the ONH. The present study's Pearson's correlation analysis and multiple regression analysis revealed that heart rate, spherical refraction and the CAVI are factors that contribute to the skew. We also observed that the skew in the capillary area of the $\mathrm{ONH}$ increases in parallel with the increase in the CAVI. In other words, the peak of the MBR - as an indicator of the velocity of the erythrocytes - in the cardiac cycle moves leftward in parallel with the exacerbation of the CAVI.

Our multiple regression analysis revealed that heart rate, the CAVI, UAC, mean IMT, spherical refraction, $\mathrm{BMI}$ and pulse pressure were factors contributing to the BOT. The BOT in the capillary area of the ONH decreased in parallel with the increase in the CAVI, UAC, mean IMT and pulse pressure. The BOT in the capillary area of the $\mathrm{ONH}$ was significantly correlated with these parameters, as was BOT in the entire $\mathrm{ONH}$, as we reported previously $[17,18,30,31]$. We reported that the $\mathrm{BOT}$ in the entire $\mathrm{ONH}$ is significantly negatively correlated with the absolute value of systemic vascular resistance [32]. We thus suggested that exacerbation of the CAVI, UAC and mean IMT - which indicates systemic arteriosclerosis - affects the sclerosis of the microcirculation in the capillaries of the $\mathrm{ONH}$. The heart rate and CAVI in particular were revealed as factors contributing to both the skew and BOT. These results suggested that large arterial function, which is manifested in the CAVI, contributes to smooth hemodynamics of microcirculation, which is shown by the LSFG. In addition, our findings indicated that the CAVI might be a more important factor contributing to the skew and BOT in the capillary area of the $\mathrm{ONH}$ than the carotid arterial thickness, kidney function and left ventricular diastolic function.

Several studies have reported that the CAVI increases not only with age but also with arteriosclerotic diseases, such as coronary artery disease, carotid arteriosclerosis, chronic kidney disease, and cerebrovascular disease [33-35]. Our prior study revealed that the CAVI is an independent risk factor for exudative age-related macular degeneration, which is a retinal and choroidal vascular disease [36]. In addition, it is possible that cardiovascular disease and cerebrovascular diseases are associated with normal tension glaucoma [37, 38]. It was also reported that ischemic changes in brain magnetic resonance imaging are more common in patients with normal-tension glaucoma than in control subjects [38, 39]. It is thus clear that important risk factors for cardiovascular disease, cerebrovascular disease and normaltension glaucoma overlap. As overlapping risk factors, we speculated in previous studies that the skew and 
BOT in the capillary area of the $\mathrm{ONH}$ are risk factors for normal-tension glaucoma and silent brain infarction $[19,20]$.

Our present findings also revealed that the systemic arterial function shown by the CAVI is one of the important contributors to ocular microcirculation. In other words, the microcirculation of the capillary area of the ONH calculated by LSFG provides a window for detecting the conditions of systemic large arteries. We believe our findings may be clues to the relationships between cardiovascular and cerebrovascular disease and ocular diseases, such as normal-tension glaucoma and retinal and choroidal vascular diseases.

In the present study, BMI was significantly positively correlated and spherical refraction was negatively correlated with BOT. These results provide new knowledge, and further detailed evaluations are needed to confirm these results. In any case, BMI and spherical refraction should be considered when interpreting blood flow data using LSFG. We excluded them from the multiple regression analysis, because the CAVI was strongly correlated with age $(r>0.60, p<0.0001)$.

Our study has some severe limitations. First, only men were included in the study, and our findings should not be generalized to women. Second, the optic-nerve head has double blood circulation; one depends on branches of the central retinal artery while the other depends on the branches of the short posterior ciliary arteries. Both circulation routes function at different perfusion pressures [40]. We evaluated these two differences in arterial blood flow of the ONH calculated by LSFG. It is known that the arteries supplying the eye have relatively high blood pressure. The pressure drop from the small arteries and arterioles to the capillaries is greater in the choroid than in the retina [41]. At the origin of the retina and ciliary arteries, blood pressure is about $65-70 \mathrm{mmHg}$. There is a direct relationship between vessel calibre and pressure of intraocular vessels [40]. Thus, another method for evaluating these two blood flows separately could need more strict research. Third, because the design of the study was crosssectional, we did not investigate whether the skew and BOT changed simultaneously with the changes in the CAVI. A prospective study should evaluate whether these parameters can detect changes in the CAVI. Further careful validation studies with larger patient populations of both genders are needed to evaluate whether these ocular microcirculation parameters obtained using LSFG could be used as novel markers for systemic large arterial function.

In conclusion, the CAVI was revealed as an independent factor contributing to both the skew and BOT in the capillary area of the ONH. Our study clarified that large arterial function shown by the CAVI contributes to smooth hemodynamics of microcirculation, which is shown by LSFG.
Acknowledgments Design and conduct of this study: T.S. and M.T. Collection, management, analysis, and interpretation of the data: T.S. and M.T. Preparation, review, or approval of the manuscript: T.S., M.T., T.M., K.S. and H.Y. We had no statistical consultation or assistance.

Compliance with ethical standards All procedures performed in studies involving human participants were in accordance with the institutional review board of Toho University Sakura Medical Center (No. 2011-009) and with the 1964 Helsinki declaration. Informed consent was obtained from all individual participants included in the study.

Funding There was no sponsorship or other support for this study.

Conflict of interest All authors certify that they have no affiliations with or involvement in any organization or entity with any financial interest (such as honoraria; educational grants; participation in speakers' bureaus; membership, employment, consultancies, stock ownership).

Open Access This article is distributed under the terms of the Creative Commons Attribution 4.0 International License (http:// creativecommons.org/licenses/by/4.0/), which permits unrestricted use, distribution, and reproduction in any medium, provided you give appropriate credit to the original author(s) and the source, provide a link to the Creative Commons license, and indicate if changes were made.

\section{References}

1. Murray CJ, Lopez AD (1997) Alternative projections of mortality and disability by cause 1990-2020: Global Burden of Disease Study. Lancet 349:1498-1504

2. Shirai K, Utino J, Otsuka K, Takata M (2006) A novel blood pressure-independent arterial wall stiffness parameter; cardioankle vascular index (CAVI). J Atheroscler Thromb 13:101-107

3. Hayashi G, Sato M, Niimi H, Handa H, Moritake K, Okumura (1975) Analysis of vascular wall constitutive law with finite deformation theory. Med Electron Biol Eng 13:293-297

4. Wong TY, Larsen EK, Klein R, Mitchell P, Couper DJ, Klein BE, Hubbard LD, Siscovick DS, Sharrett AR (2005) Cardiovascular risk factors for retinal vein occlusion and arteriolar emboli: the atherosclerosis risk in communities \& cardiovascular health studies. Ophthalmology 112:540-547

5. Newman-Casey PA, Stem M, Talwar N, Musch DC, Besirli CG, Stein JD (2014) Risk factors associated with developing branch retinal vein occlusion among enrollees in a United States managed care plan. Ophthalmology 121:1939-1948

6. Kaderli AA, Kaderli B, Gullulu S, Avci R (2010) Impaired aortic stiffness and pulse wave velocity in patients with branch retinal vein occlusion. Graefes Arch Clin Exp Ophthalmol 248:369-374

7. Taniguchi H, Shiba T, Maeno T, Takahashi M (2015) Evaluation of carotid atherosclerosis, peripheral arterial disease, and chronic kidney disease in patients with exudative age-related macular degeneration without coronary artery disease or stroke. Ophthalmologica 233:128-133

8. Klein R, Cruickshanks KJ, Myers CE, Sivakumaran TA, Iyengar SK, Meuer SM, Schubert CR, Gangnon RE, Klein BE (2013) The relationship of atherosclerosis to the 10 -year cumulative incidence of age-related macular degeneration: the Beaver Dam studies. Ophthalmology 120:1012-1019

9. Olea JL, Tuñón J (2012) Patients with neovascular age-related macular degeneration in Spain display a high cardiovascular risk. Eur J Ophthalmol 22:404-411 
10. Liew G, Wang JJ (2011) Retinal vascular signs: a window to the heart? Rev Esp Cardiol 64:515-521

11. Tamaki Y, Araie M, Kawamoto E, Eguchi S, Fujii H (1995) Noncontact, two-dimensional measurement of tissue circulation in choroid and optic nerve head using laser speckle phenomenon. Exp Eye Res 60:373-383

12. Isono H, Kishi S, Kimura Y, Hagiwara N, Konishi N, Fujii H (2003) Observation of choroidal circulation using index of erythrocytic velocity. Arch Ophthalmol 121:225-231

13. Fujii H (1994) Visualisation of retinal blood flow by laser speckle flow-graphy. Med Biol Eng Comput 32:302-304

14. Fujii H (2000) Laser speckle flowgraphy. In: Medical diagnostic techniques and procedures. Narosa Publishing House, New Delhi, London, pp 216-224

15. Takahashi H, Sugiyama T, Tokushige H, Maeno T, Nakazawa T, Ikeda T, Araie M (2013) Comparison of CCD-equipped laser speckle flowgraphy with hydrogen gas clearance method in the measurement of optic nerve head microcirculation in rabbits. Exp Eye Res 108:10-15

16. Sugiyama T (2014) Basic technology and clinical applications of the updated model of laser speckle flowgraphy to ocular diseases. Photonics 1:220-234

17. Shiba T, Takahashi M, Hori Y, Maeno T (2012) Pulse-wave analysis of optic nerve head circulation is significantly correlated with brachial-ankle pulse-wave velocity, carotid intima-media thickness, and age. Graefes Arch Clin Exp Ophthalmol 250:1275-1281

18. Shiba T, Takahashi M, Hori Y, Maeno T, Shirai K (2012) Optic nerve head circulation determined by pulse wave analysis is significantly correlated with cardio-ankle vascular index, left ventricular diastolic function, and age. J Atheroscler Thromb 19:999-1005

19. Shiga Y, Omodaka K, Kunikata H, Ryu M, Yokoyama Y, Tsuda S, Asano T, Maekawa S, Maruyama K, Nakazawa T (2013) Waveform analysis of ocular blood flow and the early detection of normal tension glaucoma. Invest Ophthalmol Vis Sci 54:7699-7706

20. Kunikata H, Aizawa N, Kudo M, Mugikura S, Nitta F, Morimoto R, Iwakura Y, Ono Y, Satoh F, Takahashi H, Ito S, Takahashi S, Nakazawa T (2015) Relationship of ocular microcirculation, measured by laser speckle flowgraphy, and silent brain infarction in primary aldosteronism. PLoS ONE 10, e0117452

21. Lee SH, Choi S, Chung WJ, Byun YS, Ryu SK, Pyun WB, Rim SJ (2008) Tissue Doppler index, E/E', and ischemic stroke in patients with atrial fibrillation and preserved left ventricular ejection fraction. J Neurol Sci 271:148-152

22. Nagueh SF, Appleton CP, Gillebert TC, Marino PN, Oh JK, Smiseth OA, Waggoner AD, Flachskampf FA, Pellikka PA, Evangelisa A (2009) Recommendations for the evaluation of left ventricular diastolic function by echocardiography. Eur J Echocardiogr 10:165-193

23. Ommen SR, Nishimura RA, Appleton CP, Miller FA, Oh JK, Redfield MM, Tajik AJ (2000) Clinical utility of Doppler echocardiography and tissue Doppler imaging in the estimation of left ventricular filling pressures: a comparative simultaneous Dopplercatheterization study. Circulation 102:1788-1794

24. O'Leary DH, Polak JF, Kronmal RA, Manolio TA, Burke GL, Wolfson SK Jr (1999) Carotid artery intima and media thickness as a risk factor for myocardial infarction and stroke in older adults. N Engl J Med 340:14-22

25. Takiuchi S, Kamide K, Miwa Y, Tomiyama M, Yoshii M, Matayoshi T, Horio T, Kawano Y (2004) Diagnostic value of carotid intima-media thickness and plaque score for predicting target organ damage in patients with essential hypertension. J Hum Hypertens 18:17-23
26. Sugiyama T, Araie M, Riva CE, Schmetterer L, Orgul S (2010) Use of laser speckle flowgraphy in ocular blood flow research. Acta Ophthalmol 88:723-729

27. Tsuda S, Kunikata H, Shimura M, Aizawa N, Omodaka K, Shiga Y, Yasuda M, Yokoyama Y, Nakazawa T (2014) Pulse-wave analysis of normal population using laser speckle flowgraphy. Curr Eye Res 39:1207-1215

28. Yanagida K, Iwase T, Yamamoto K, Ra E, Kaneko H, Murotani K, Matsui S, Terasaki H (2015) Sex-related differences in ocular blood flow of healthy subjects using laser speckle flowgraphy. Invest Ophthalmol Vis Sci 56:4880-4890

29. Matthews DR, Hosker JP, Rudenski AS, Naylor BA, Treacher DF, Turner RC (1985) Homeostasis model assessment: insulin resistance and beta-cell function from fasting plasma glucose and insulin concentrations in man. Diabetologia 28:412-419

30. Rina M, Shiba T, Takahashi M, Hori Y, Maeno T (2015) Pulse waveform analysis of optic nerve head circulation for predicting carotid atherosclerotic changes. Graefes Arch Clin Exp Ophthalmol 253:2285-2291

31. Shiba T, Takahashi M, Maeno T (2014) Pulse-wave analysis of optic nerve head circulation is significantly correlated with kidney function in patients with and without chronic kidney disease. J Ophthalmol. doi:10.1155/2014/291687

32. Shiba T, Takahashi M, Hashimoto R, Matsumoto T, Hori Y (2016) Pulse waveform analysis in the optic nerve head circulation reflects systemic vascular resistance obtained via a Swan-Ganz catheter. Graefes Arch Clin Exp Ophthalmol. In press. doi:10.1007/ s00417-016-3289-y

33. Nakamura K, Tomaru T, Yamamura S, Miyashita Y, Shirai K, Noike H (2008) Cardio-ankle vascular index is a candidate predictor of coronary atherosclerosis. Circ J 72:598-604

34. Izuhara M, Shioji K, Kadota S, Baba O, Takeuchi Y, Uegaito T, Mutsuo S, Matsuda M (2008) Relationship of cardio-ankle vascular index (CAVI) to carotid and coronary arteriosclerosis. Circ J 72: 1762-1767

35. Yamamoto N, Yamanaka G, Ishikawa M, Takasugi E, Murakami S, Yamanaka T, Ishine M, Matsubayashi K, Hanafusa T, Otsuka K (2009) Cardio-ankle vascular index as a predictor of cognitive impairment in community-dwelling elderly people: four-year followup. Dement Geriatr Cogn Disord 28:153-158

36. Taniguchi H, Shiba T, Takahashi M, Kanai H, Hori Y, Shirai K, Maeno T (2013) Cardio-ankle vascular index elevation in patients with exudative age-related macular degeneration. $\mathrm{J}$ Atheroscler Thromb 20:903-910

37. Doyle A, Bensaid A, Lachkar Y (2005) Central corneal thickness and vascular risk factors in normal tension glaucoma. Acta Ophthalmol Scand 83:191-195

38. Ong K, Farinelli A, Billson F, Houang M, Stern M (1995) Comparative study of brain magnetic resonance imaging findings in patients with low-tension glaucoma and control subjects. Ophthalmology 102:1632-1638

39. Stroman GA, Stewart WC, Golnik KC, Cure J, Olinger RE (1995) Magnetic resonance imaging in patients with low-tension glaucoma. Arch Ophthalmol 113:168-172

40. Schmidl D, Boltz A, Kaya S, Werkmeister R, Dragostinoff N, Lasta M, Polska E, Garhöfer G, Schmetterer L (2012) Comparison of choroidal and optic nerve head blood flow regulation during changes in ocular perfusion pressure. Invest Ophthalmol Vis Sci 53: 4337-4346

41. Nagaoka T, Sato E, Takahashi A, Yokota H, Sogawa K, Yoshida A (2010) Impaired retinal circulation in patients with type 2 diabetes mellitus: retinal laser Doppler velocimetry study. Invest Ophthalmol Vis Sci 51:6729-6734 